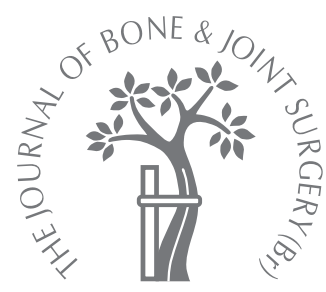

\title{
The effect of a neck-retaining femoral implant on leg-length inequality following total hip arthroplasty
}

\author{
A RADIOLOGICAL STUDY
}

\begin{abstract}
V. Khanduja, V. Tek, G. Scott
\end{abstract}

From the Bone and Joint Research Unit, Royal London Hospital, London, England

V. Khanduja, MBBS, FRCS(Tr \& Orth), MSc, Specialist Registrar

V. Tek, MRCS, Clinical Fellow - G. Scott, FRCS, Consultant Orthopaedic Surgeon

Bone \& Joint Research Unit The Royal London Hospital, Whitechapel, London E1 1BB, UK.

Correspondence should be sent to Mr V. Khanduja at 109a Queens Avenue, Watford, Hertfordshire WD18 7NU, UK; e-mail:

vikaskhanduja@aol.com

(C)2006 British Editorial Society of Bone and Joint Surgery doi:10.1302/0301-620X.88B6. $17190 \$ 2.00$

$J$ Bone Joint Surg [Br] 2006;88-B:712-15.

Received 14 September 2005; Accepted after revision 25 January 2006

\begin{abstract}
The aim of this study was to assess whether a femoral component which retained the neck reduced the incidence of leg-length inequality following total hip arthroplasty. A retrospective review was undertaken of 130 consecutive primary total hip arthroplasties performed between April 1996 and April 2004 using such an implant. There were 102 suitable patients for the study. Standardised pre- and post-operative pelvic radiographs were measured by an independent investigator to the nearest millimetre.

The leg-length inequality was reduced from a mean pre-operative value of $-0.71 \mathrm{~cm}$ to a mean of $0.11 \mathrm{~cm}$ post-operatively. Of the 102 patients $24(23.5 \%)$ had an equal leg-length post-operatively, and $95(93.1 \%)$ had a leg-length inequality between $-1 \mathrm{~cm}$ and $1 \mathrm{~cm}$.
\end{abstract}

Inequality of leg length is a recognised complication following total hip arthroplasty (THA). ${ }^{1}$ The incidence has been reported to range from $1 \%$ to $27 \%$ with the mean varying from $1 \mathrm{~mm}$ to $15.9 \mathrm{~mm}$ in some series. ${ }^{2-4}$ Minor discrepancies of up to $1 \mathrm{~cm}$ are usually well tolerated, but in some patients even a small disparity may be a source of dissatisfaction. $^{5}$

Although absolute equalisation of leg length is difficult to achieve after THA, the discrepancy can be minimised by a series of steps taken both before and during the operation. The literature is replete with articles describing methods to measure leg-length inequality and most techniques can be grouped into one of three categories; ${ }^{6-8} 1$ ) those which rely on preoperative templating and determination of the osteotomy site with intra-operative determination of the osteotomy site in relation to the lesser trochanter; 2) those using intra-operative pelvic and femoral markers for reference and; 3) experimental methods which employ complex mathematical calculations and ultrasound probes to measure leg lengths accurately. However, little has been written as to whether the design of an implant can help reduce leg-length inequality. This study has assessed leg-length inequality in patients undergoing THA using a femoral component which allows retention of the neck.

\section{Patients and Methods}

A total of 130 consecutive patients who had a unilateral primary total hip arthroplasty in our unit between April 1996 and April 2004 were included in the study. We excluded 28 patients because of anatomical anomalies in the proximal femur or acetabulum, advanced erosive arthritis, collapse of the femoral head or acetabular erosion in the contralateral hip, since in these circumstances equalisation of the leg length was not likely to be achieved. None of the remaining 102 patients had undergone previous surgery to the hip, and the contralateral hip was normal or near normal. The assessment of leg-length inequality employed in this study required the opposite hip to be normal with an intact Shenton's line.

All the patients had undergone total hip arthroplasty using a Freeman neck-retaining femoral component (Finsbury Instruments Ltd, Leatherhead, United Kingdom) implanted via a modified anterolateral approach. ${ }^{9}$ Both the cemented and the hydroxyapatite (HA)coated version of the stem were included in the study.

All the operations were performed by the senior author (GS) or by trainees under his direct supervision. The intention was to restore or retain an intact Shenton's line. Radiographic templating to define the location of the proximal femoral resection was not carried out in advance. Because the neck was retained, the relationship of the femoral part of Shenton's line and its pelvic counterpart could be identified intra-operatively. With the patient in the lateral position and inclined backwards by approximately $30^{\circ}$, the hip joint was exposed via a modified anterolateral approach described 


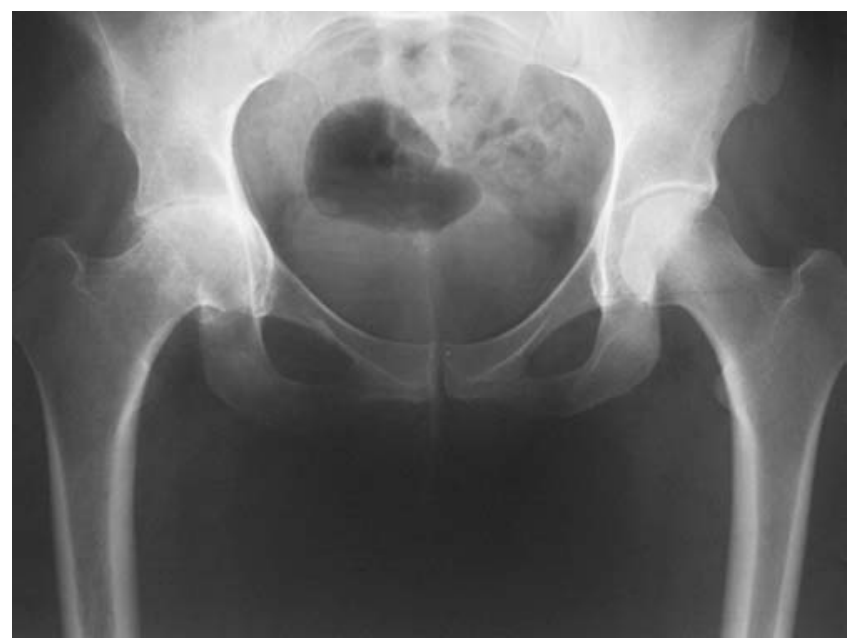

Fig. 1

Pre-operative radiograph with osteoarthritis of the right hip.

by Stephenson and Freeman in $1991 .{ }^{9}$ The surgical technique employed has been previously described. ${ }^{10}$ The femur is prepared first. Osteophytes are trimmed from the head/ neck junction, particularly medially as this represents the femoral side of Shenton's line. The femoral head is resected just above the head/neck junction. The femoral canal is reamed and the interior of the neck shaped for the prosthesis by a slot cutter, which is advanced to preserve a minimum thickness of $5 \mathrm{~mm}$ of bone at the lower border of the neck to surround the prosthesis. With the slot cutter at the correct position the residual portion of the femoral head is removed flush with the cutter, representing the position for a medium neck length prosthesis. The correct diameter femoral trial is then introduced into the reamed cavity, where it should lie recessed by about $5 \mathrm{~mm}$. The acetabulum is then prepared. Next, a $6.5 \mathrm{~mm}$ indicator rod is placed at the teardrop to mark the pelvic side of Shenton's line. Either a short or a medium modular trial trunnion is added to the femoral trial, to which one of five lengths of modular trial femoral head is added. The hip with the trials in place is then relocated. The hip is held so that it is fully extended and in neutral position. The indicator rod is placed so that it is perpendicular to the long axis of the body, i.e. it is orientated as a continuation of the inter-teardrop line. The intersection of the upper margin of the rod with the retained femoral neck can then be viewed. Using the correct combination of trial femoral heads and femoral component neck lengths, a situation is reached where Shenton's line can be judged intact. The combination of trial components then represents the size combination for the definitive implants. If the required combination includes a femoral component with a short neck length, the $5 \mathrm{~mm}$ of bone protruding beyond the femoral trial is removed before the latter is withdrawn. Definitive components can then be implanted.

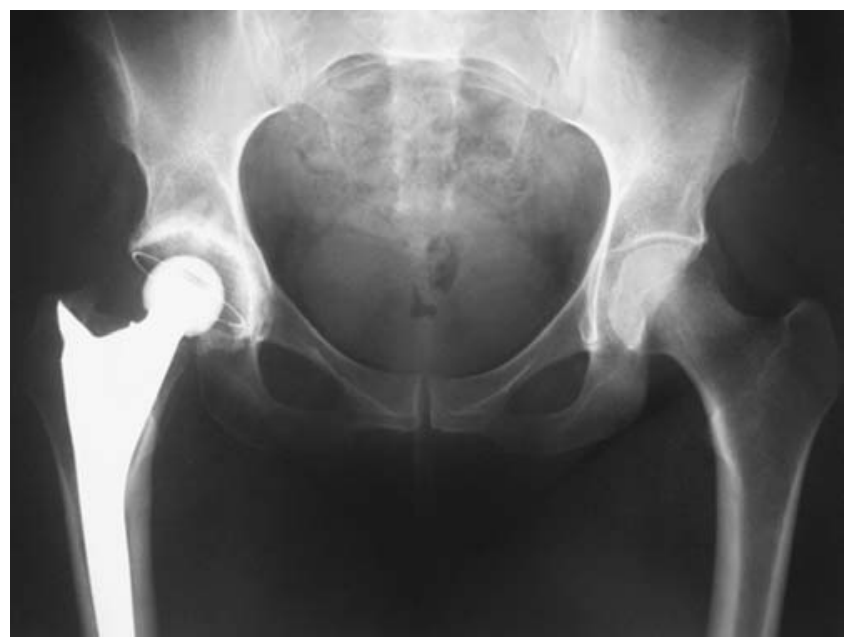

Fig. 2

Post-operative radiograph with a neck-retaining femoral implant.

Radiological assessment. Anteroposterior radiographs of the pelvis were obtained pre- and post-operatively in a standardised fashion with both hips extended and internally rotated, as in previous studies ${ }^{11,12}$ (Figs 1 and 2). Leg-length inequality was assessed using the method described by Woolson et al. ${ }^{8}$ The pelvic reference point was defined by drawing a transverse line through the inferior border of the teardrops. The most prominent point on the lesser trochanter on each side was taken as the femoral reference point. A line was drawn from each femoral reference point to the perpendicular intersection with the pelvic reference line. This distance between the two reference points was taken as an index of pre- and post-operative leg length. This method does not take into account other discrepancies of length in the lower limb due to changes remote from the hip, but does give an accurate assessment of the situation before and after surgery. It has been used reliably in previous studies. ${ }^{12}$

All the measurements were made by a single investigator (VK) who was not involved in the operations, using a ruler and recorded to the nearest millimetre. Of the 102 radiographs 20 were re-measured by the same investigator after shuffling the films to calculate the intra-observer variation. This revealed an accuracy of SD $1 \mathrm{~mm}$. Magnification was determined by measuring the diameter of the femoral head on the film and then dividing it by the known diameter of the prosthetic head used. This gave an average magnification of $18 \%$.

Leg length inequality was defined as equal, long or short when compared with the non-operated side.

\section{Results}

The radiographs of 102 patients ( 58 men and 44 women) were assessed. Of these, 65 had a cemented femoral implant and 37 had a hydroxyapatite-coated device. 
Table I. Pre-operative leg lengths

\begin{tabular}{|c|c|}
\hline Leg length* & Numbe \\
\hline Long & 14 \\
\hline Short & 81 \\
\hline Equal & 7 \\
\hline
\end{tabular}

* the groups long, short and equal are made in comparison with the unaffected normal side
Table II. Post-operative leg lengths

\begin{tabular}{ll}
\hline Leg length & Number (\%) \\
\hline Long & $49(48)$ \\
Short & $29(28.5)$ \\
Equal & $24(23.5)$ \\
\hline * the groups long, short and equal \\
are made in comparison with the
\end{tabular}
unaffected normal side

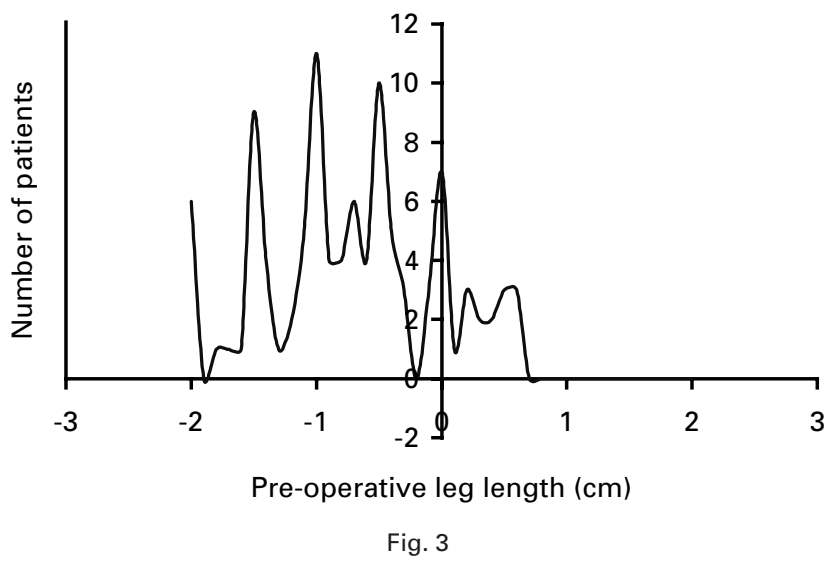

Graph showing the spread of leg lengths pre-operatively.

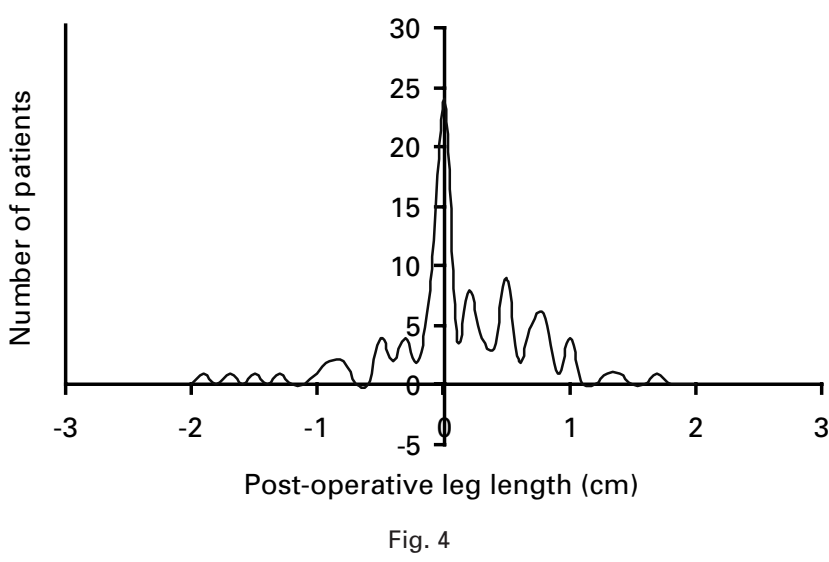

Graph showing the spread of leg lengths post-operatively.
The pre- and post-operative leg lengths are shown in Tables I and II respectively. The mean pre-operative leglength inequality was $-0.71 \mathrm{~cm}(0.6$ to -2$)$ and postoperatively the mean leg-length inequality was $0.11 \mathrm{~cm}(1.7$ to -2$)$.

Of the 102 patients, $24(23.5 \%)$ achieved equal leg lengths following THA, 49 (48\%) limbs were lengthened and $29(28.5 \%)$ remained short. Of the 102 THAs 95 $(93.1 \%)$ had leg lengths equalised to within $-1 \mathrm{~cm}$ to $+1 \mathrm{~cm}$, and $72(70.5 \%)$ were within a range of $-0.5 \mathrm{~cm}$ to $+0.5 \mathrm{~cm}$. The pre- and post-operative distributions of leg-length inequality are depicted in Figures 3 and 4, respectively.

\section{Discussion}

Total hip arthroplasty has revolutionised the treatment of end-stage arthritis of the hip and is among the most costeffective of all medical interventions. ${ }^{13}$ The high success rate, increasing age of the population and ever-increasing indications have led to a larger number of hip arthroplasties being performed worldwide. Inevitably, as the number of THAs increases so do the complications, but these can be minimised by adhering to the biomechanical principles of a successful hip arthroplasty, namely a correct centre of rotation, correct orientation of the components, adequate offset and equal leg lengths.
Much importance is placed on achieving the correct centre of rotation, correct component orientation and an adequate offset, but the need to achieve equal leg lengths is emphasised less. Minor discrepancies, of less than $1 \mathrm{~cm}$, are usually well tolerated, ${ }^{11,14}$ but inequalities of more than $1 \mathrm{~cm}$ can be associated with a risk of dislocation, low back pain, functional scoliosis, sciatic nerve palsy, pelvic tilt, altered gait, patient dissatisfaction and litigation. ${ }^{15-18}$ Functional outcome and leg-length inequality following THA appear to be correlated. ${ }^{19}$ Konyves and Bannister ${ }^{19}$ observed that $56(62 \%)$ of the 90 limbs in their study were longer by a mean of $9 \mathrm{~mm}$ after THA; and this difference in leg length was perceived by 24 patients (43\%). In addition, the mean Oxford Hip Score was worse in the patients with unequal leg lengths. ${ }^{19}$ In another study by Edeen et $\mathrm{al}^{5} 68$ patients were interviewed and examined after THA, $32 \%$ of whom were aware of a leg-length inequality with the average being $14.9 \mathrm{~mm}$. More than half of these were dissatisfied with the THA because of this. ${ }^{5}$ Reduction of leg-length inequality following THA is therefore important.

The Freeman neck-retaining femoral component (Finsbury Instruments Ltd) was first implanted in 1981 with the biomechanical intention of resisting downward migration by a factor of 3 , reducing the varus turning moment by a 
factor of 4 and reducing the torsional moment by increasing the area of proximal bone contact. ${ }^{20,21}$

A further advantage of retaining the femoral neck is the opportunity to assess the integrity of Shenton's line intraoperatively and to reduce the possibility of leg-length inequality. Although equal leg lengths were only obtained in a quarter of the cases in this study, in $93 \%$ of the patients (95) the leg length was restored to within -1 to $+1 \mathrm{~cm}$ of equality.

Compared to other studies specifically assessing leglength inequality following THA our results are better in terms of the extent of leg-length inequality and the number of patients achieving equal leg lengths. ${ }^{2,5,7}$ The only study with superior results to ours was by Woolson et $\mathrm{al}^{8}{ }^{8}$ in which $97 \%$ of the patients had a leg-length inequality of less than $1 \mathrm{~cm}$. Woolson's ${ }^{8}$ method consists of exact positioning of the femoral neck osteotomy from calculations made from pre-operative planning with overlay templates. The method assumes that the same amount of femoral head, neck and remaining acetabular cartilage removed during the procedure can be replaced with a matching amount of prosthetic material and thus the leg lengths should remain equal. ${ }^{8}$

Even with accurate pre-operative templating the surgeon still needs to measure limb lengths intra-operatively. Adjustments are often made by subjective evaluation of tension of the abductor muscles when the patient is relaxed by the anaesthesia and the muscles divided. This is not precise and can lead to an increase in the distance between the pelvis and the femur, resulting in leg length inequality. Referencing from Shenton's line in the presence of the retained femoral neck converts this subjective evaluation into a more precise procedure, improving the equalisation of the leg lengths.

We believe our results demonstrate that the Freeman neck-retaining femoral component (Finsbury Instruments Ltd) recreates the anatomy of the proximal femur, thereby minimising leg length inequality following THA.

The authors would like to thank Mrs Judy Houghton for her secretarial help during the preparation of this manuscript.
Although none of the authors has received or will receive benefits for personal or professional use from a commercial party related directly or indirectly to the subject of this article, benefits have been or will be received but will be directed solely to a research fund, foundation, educational institution, or other nonprofit organisation with which one or more of the authors are associated.

\section{References}

1. Williamson JA, Reckling FW. Limb length discrepancy and related problems following total hip joint replacement. Clin Orthop 1978;134:135-8.

2. Bose WJ. Accurate limb-length equalization during total hip arthroplasty. Orthopedics 2000;23:433-6.

3. Ranawat CS, Rodriguez JA. Functional leg-length inequality following total hip arthroplasty. J Arthroplasty 1997;12:359-64.

4. Turula KB, Friberg $\mathbf{0}$, Lindholm S, Tallroth K, Vankka E. Leg length inequality after total hip arthroplasty. Clin Orthop 1986;202:163-8.

5. Edeen J, Sharkey PF, Alexander AH. Clinical significance of leg-length inequality after total hip arthroplasty. Am J Orthop 1995;24:347-51.

6. Eggli S, Psian M, Muller ME. The value of preoperative planning for total hip arthroplasty. J Bone Joint Surg [Br] 1998;80-B:382-90.

7. Ranawat CS. The pants too short, the leg too long! Orthopedics 1999:22:845-6.

8. Woolson ST, Hartford JM, Sawyer A. Results of a method of leg-length equalization for patients undergoing primary total hip replacement. J Arthroplasty 1999;14:159-64.

9. Stephenson PK, Freeman MA. Exposure of the hip using a modified anterolateral approach. J Arthroplasty 1991;6:137-45.

10. Albrektsson BEJ, Freeman MAR, Carlsson LV, Tuke MA, Clarke JM. Retention of the femoral neck: a method to enhance prosthetic fixation. J Orthop Surgical Techniques 1987;3:99-124.

11. Sarangi PP, Bannister GC. Leg length discrepancy after total hip replacement. Hip 1997;7:121-4.

12. White TO, Dougall TW. Arthroplasty of the hip: leg length is not important. J Bone Joint Surg [Br] 2002;84-B:335-8.

13. Laupacis A, Bourne R, Rorabeck C, et al. The effect of elective total hip replacement on health-related quality of life. J Bone Joint Surg [Am]1993;75-A:1619-26.

14. Love BRT, Wright $\mathbf{K}$. Leg length discrepancy after total hip replacement. J Bone Joint Surg [Br] 1983;65-B:103 (abstract).

15. Friberg $\mathbf{0}$. Clinical symptoms and biomechanics of lumbar spine and hip joint in leg length inequality. Spine 1983;8:643-51.

16. Friberg $\mathbf{0}$. Biomechanical significance of the correct length of lower limb prostheses: a clinical and radiological study. Prosthet Orthot Int 1984;8:124-9.

17. Lai KA, Lin CJ, Jou IM, Su FC. Gait analysis after total hip arthroplasty with leglength equalization in women with unilateral congenital complete dislocation of the hip: comparison with untreated patients. J Orthop Res 2001;19:1147-52.

18. Rosler J, Perka C. The effect of anatomical positional relationships on kinetic parameters after total hip replacement. Int Orthop 2000;24:23-7.

19. Konyves A, Bannister GC. The importance of leg length discrepancy after total hip arthroplasty. J Bone Joint Surg [Br] 2005;87-B:155-7.

20. Freeman MA. Why resect the neck? J Bone Joint Surg [Br] 1986;68-B:346-9.

21. Carlsson L, Albrektsson B, Freeman MA. Femoral neck retention in hip arthroplasty: a cadaver study of mechanical effects. Acta Orthop Scand 1988;59:6-8. 